The use of buffer pellets to pseudo hot seed (RE)-Ba-Cu-O-(Ag) single grain bulk superconductors

This content has been downloaded from IOPscience. Please scroll down to see the full text.

2016 Supercond. Sci. Technol. 29015010

(http://iopscience.iop.org/0953-2048/29/1/015010)

View the table of contents for this issue, or go to the journal homepage for more

Download details:

IP Address: 131.111.184.102

This content was downloaded on 07/12/2015 at 10:47

Please note that terms and conditions apply. 


\title{
The use of buffer pellets to pseudo hot seed (RE)-Ba-Cu-O-(Ag) single grain bulk superconductors
}

\author{
Yunhua Shi, Devendra Kumar Namburi, Wen Zhao, John H Durrell, \\ Anthony R Dennis and David A Cardwell
}

Department of Engineering, University of Cambridge, Cambridge CB2 1PZ, UK

E-mail: ys206@cam.ac.uk

Received 29 July 2015, revised 19 September 2015

Accepted for publication 28 September 2015

Published 1 December 2015

\begin{abstract}
Reliable seeding of the superconducting (RE) $\mathrm{Ba}_{2} \mathrm{Cu}_{3} \mathrm{O}_{7-\delta}(\mathrm{RE}-123)$ phase is a critical step in the melt growth of large, single grain, (RE)BaCuO ((RE)BCO) bulk superconductors. Recent improvements to the top seeded melt growth (TSMG) processing technique, which is an established method of fabricating bulk (RE)BCO superconductors, based on the use of a buffer layer between the seed and green body preform, has significantly improved the reliability of the single grain growth process. This technique has been used successfully for the primary TSMG and infiltration melt growth of all compositions within the ((RE)BCO-Ag) family of materials (where $\mathrm{RE}=\mathrm{Sm}, \mathrm{Gd}$ and $\mathrm{Y}$ ), and in recycling processes. However, the mechanism behind the improved reliability of the melt process is not understood fully and its effect on the superconducting properties of the fully processed single grains is not clear. In this paper, we investigate the effect of the use of a buffer pellet between the seed and green body on the microstructure, critical current, critical temperature and trapped field of the bulk superconductor. We conclude that the introduction of the buffer pellet evolves the melt growth process towards that observed in the technologically challenging hot seeding technique, but has the potential to yield high quality single grain samples but by a commercially viable melt process.
\end{abstract}

Keywords: buffer, YBCO single grains, hot seeding

\section{Introduction}

Bulk, single grain superconductors in the (RE)-Ba-Cu-O-Ag $((\mathrm{RE}) \mathrm{BCO}, \mathrm{RE}=\mathrm{Nd}, \mathrm{Sm}, \mathrm{Eu}, \mathrm{Gd}, \mathrm{Y}, \mathrm{Yb})$ family of materials have considerable potential for practical applications due to their ability to trap magnetic fields up to ten times higher than the fields available using conventional permanent magnets [1-3]. Indeed, a world record trapped field of $17.6 \mathrm{~T}$ has been obtained at $26 \mathrm{~K}$ at the centre of a stack of two GdBCOAg single grain bulk superconductors of diameter $24 \mathrm{~mm}$ [4].

Single grain bulk (RE)BCO superconductors have a textured lattice structure that effectively overcomes the 'weak-link' [5] problem associated with randomly orientated grains within the (RE)BCO bulk microstructure. This makes it possible for large, persistent super-current to flow throughout the single grain bulk sample, which consequently results in the trapping of large magnetic fields. Unfortunately, the process required to fabricate a single grain bulk sample is relatively complex. For example, the commonly used top seeded melt growth (TSMG) [6] process consists of the preparation of precursor powder, the selection of a seed, control of the seeding process itself, single grain nucleation and growth via a controlled peritectic reaction $\left[\mathrm{RE}_{2} \mathrm{Ba}_{1} \mathrm{Cu}_{1} \mathrm{O}_{5}\right.$ (RE-211) $+\mathrm{Ba}_{3} \mathrm{Cu}_{5} \mathrm{O}_{8} \quad$ (liquid) $\rightarrow \mathrm{RE}_{1} \mathrm{Ba}_{2} \mathrm{Cu}_{3} \mathrm{O}_{6.5} \quad$ (RE123)] during slow cooling from $1000{ }^{\circ} \mathrm{C}$ and, finally, post melt-processing oxygenation. While all these steps are fundamental to the single grain growth process, seeding is a particularly crucial step and is the most common mechanism of failure of the entire TSMG process. 
The seed material must necessarily have a higher melting temperature than the material to be grown to initiate the growth of a (RE)BCO-Ag single grain in the cold seeded TSMG process. The seed must also have the same, or very similar, tetragonal lattice structure and be chemically stable during the melt process. In practice, this requires the seed to have a melting temperature that is up to $40{ }^{\circ} \mathrm{C}$ higher than the melting/peritetic temperature of the material to be grown in single grain form $\left(1050{ }^{\circ} \mathrm{C}\right.$ in the case of seeding the YBCO system). The seeding process takes place over a range of temperatures between the melting temperature $T_{\mathrm{m}}$, which is the highest temperature used in TSMG and at which peritectic decomposition occurs completely, and $T_{\mathrm{g} 1}$, which is the temperature at which slow cooling commences and at which single grain growth begins. The length of time required for an individual seed to retain its integrity (i.e. without degrading structurally or chemically) could be several hours, so, consequently, the chemical stability of the seed is essential for the reliability of the seeding process. Seeds can sometimes fail, even if the melting temperature and lattice structure conditions are satisfied, regardless of which type of seed is used (for example either SmBCO, NdBCO or a generic seed [7]). This occurs more frequently when silver is added to the precursor powder to improve the mechanical properties of the fully processed sample $[8,9]$. Silver can diffuse into the seed at elevated temperature and decrease its melting temperature by up to $40{ }^{\circ} \mathrm{C}$. As a result, this establishes a requirement for a minimum peritectic decomposition temperature of at least $40{ }^{\circ} \mathrm{C}$ greater than that of the material to be seeded. Thin film seeds provide the highest $T_{\mathrm{m}}[10,11]$, although they are generally expensive to produce compared to seeds fabricated by a bulk processing technique.

Hot-seeding [12] is a relatively complex process that involves placing a seed onto the surface of the sample to be seeded at a temperature between $T_{\mathrm{m}}$ and $T_{\mathrm{g} 1}$. However, this process does not require the seed to have a melting temperature higher than $T_{\mathrm{m}}$ and, as a result, the seeding process does not usually fail due to thermal instability. However, the hot seeding process requires a relatively complex, specially designed furnace to enable placement of the seed and is not a practical way of performing reliable, medium scale, multigrain or multi-seeding processes [13].

Several authors, including Kim et al [14], Yao et al [10, 15] and Zhou et al [16], have described a method in which a small pellet is inserted between the seed and green precursor bulk sample in order to inhibit the diffusion of harmful elements from the seed into the growing bulk sample $[14,10]$. This method may also be used to accommodate lattice mismatch between a $\mathrm{MgO}$ seed and the precursor pellet [16]. The Cambridge Bulk Superconductivity Group has employed this buffer technique further to inhibit the diffusion of $\mathrm{Ag}$ in $\mathrm{Ag}$ doped SmBCO samples into the seed, lowering its melting point and resulting in growth failure [17]. We have also applied the buffer technique to the YBCO [18] and SmBCO systems [19], where $T_{\mathrm{m}}$ is very close to the melting temperature of the seed. This technique is now frequently used in all (RE)Ba-Cu-O-(Ag) [(RE)BCO-Ag] systems (where $\mathrm{RE}=\mathrm{Sm}, \mathrm{Gd}$ and $\mathrm{Y}$ ); in primary growth [17-19]

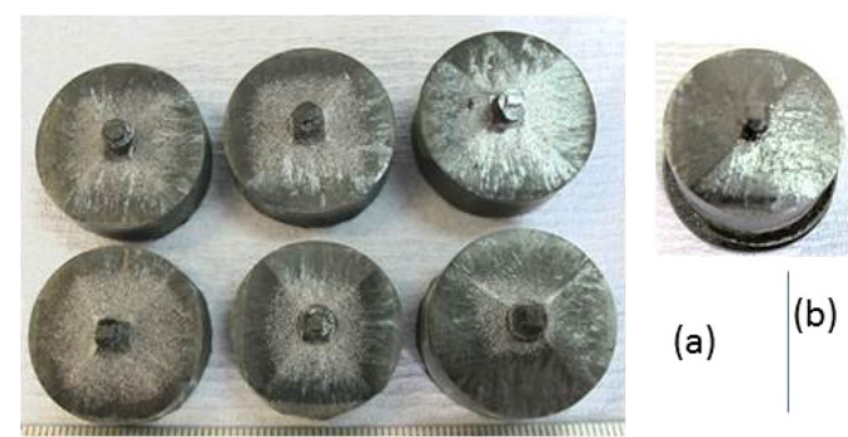

Figure 1. (a) Six GdBCO-Ag single grains grown using buffers, employing top seeded melt growth process and (b) one YBCO single grain of diameter $25 \mathrm{~mm}$ grown using the buffer-aided infiltration growth technique.

including most recent grown $\mathrm{GdBCO}-\mathrm{Ag}$ samples as shown in figure 1(a); recycling processes, where 64 (RE)BCO-(Ag) failed samples were recycled through providing adequate phase [20] and in the infiltration growth process [21]. A $25 \mathrm{~mm}$ dia.YBCO single grain grown by buffer-aided infiltration growth technique is shown in figure 1(b). These later two processes involve the generation of relatively large quantities of liquid phase during the crystal growth process, which can influence deleteriously the chemical stability of the seed.

Although the influence of buffer layers on the seeding process has been investigated comprehensively, the mechanism involved in the seeding process and its effect on the superconducting properties of the resulting single grain, rather than on the grain nucleation process itself, has not been studied systematically. It is this issue that is addressed in this paper. A set of YBCO single grains of different sizes processed with different size buffer layers were fabricated for this study and their superconducting properties, $T_{\mathrm{c}}$ and $J_{\mathrm{c}}$ and related microstructures at different positions within the parent grain were measured. Finally the trapped field, which is often the key figure of merit for engineering applications, for single grain bulk (RE)BCO samples, was measured for each sample.

We summarise the effect of the buffering technique on single grain growth and compare it to the hot seeding technique. We also examine the changes in microstructure and superconducting properties of (RE)-Ba-Cu-O- $(\mathrm{Ag})$ single grains associated with the use of a buffer layer and, finally, we discuss briefly the mechanisms involved in the growth process.

\section{Experiment}

$\mathrm{YBa}_{2} \mathrm{Cu}_{3} \mathrm{O}_{7-\delta}$ (Y-123) (99.9\% Toshima) and $\mathrm{Y}_{2} \mathrm{BaCuO}_{5}$ (Y-211) (99.9\% Toshima) powders of average particle size 2 and $1 \mu \mathrm{m}$ were mixed thoroughly with $\mathrm{CeO}_{2}(99.9 \%$, Alfa Aesar) by a tubular mill to form the precursor powder. The precursor powder, of composition $75 \mathrm{wt} \% \mathrm{Y}-123+25 \mathrm{wt} \%$ $\mathrm{Y}-211+0.5 \mathrm{wt} \% \mathrm{CeO}_{2}$, was then pressed uniaxially into pellets. Three YBCO single grains of diameter $16 \mathrm{~mm}$ (post growth) were fabricated in an air atmosphere, as shown in 


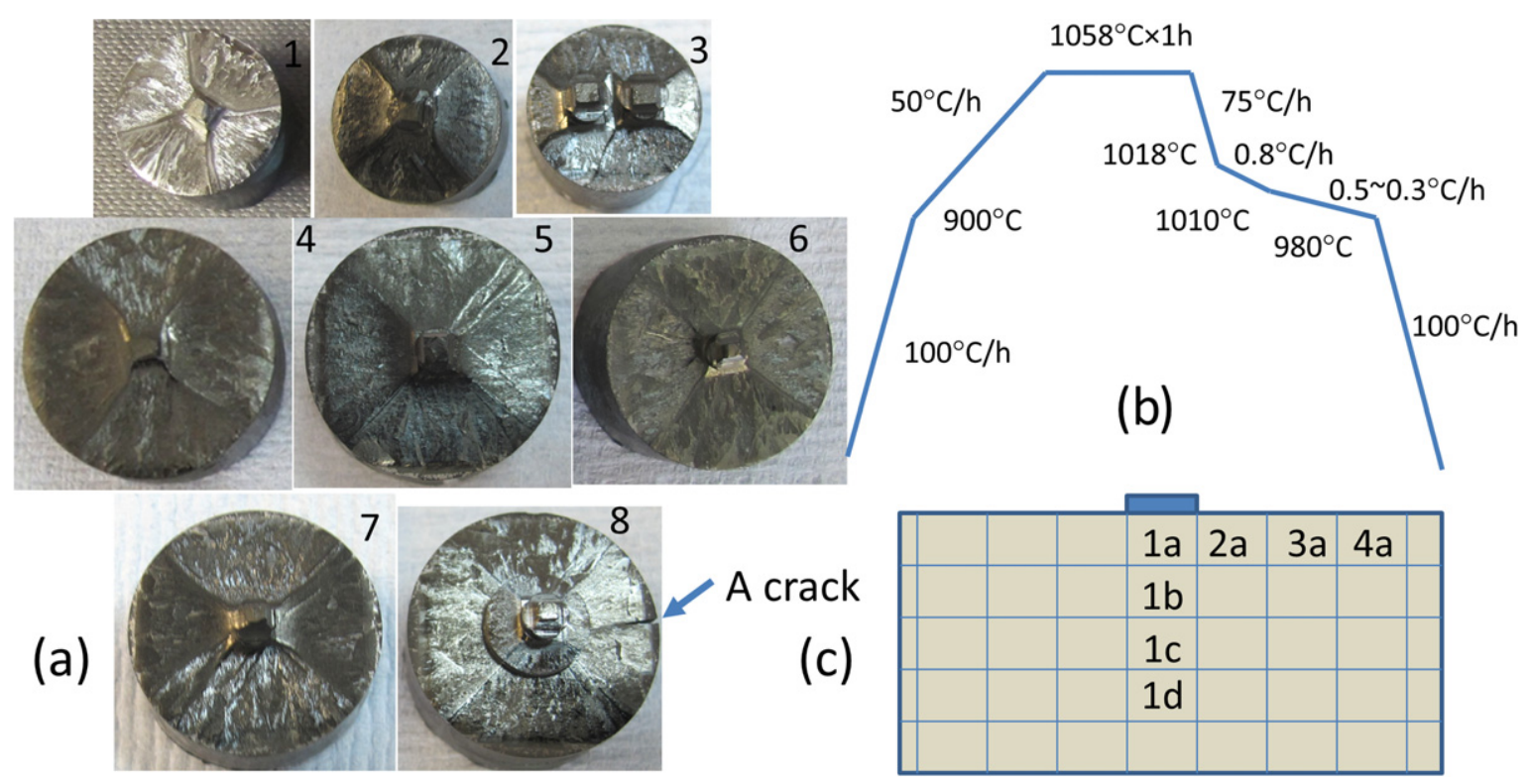

Figure 2. (a) YBCO single grains of diameter 16 and $25 \mathrm{~mm}$ fabricated using a single seed or seed and buffer layer combination. (b) Heating profiles used to melt-process the single grains. (c) A schematic diagram illustrating the positions of the sub-specimens within a cross-section of the parent single grain for the measurement of $T_{\mathrm{c}}$ and $J_{\mathrm{c}}$

figure 2(a), using the heating profile shown in figure 2(b). Sample 1 was made using a single seed, sample 2 using a single seed plus a single buffer layer of diameter $4 \mathrm{~mm}$ (post growth) and sample 3 using a pair of ' $0-0$ ' aligned seeds [22] plus two buffer layers of diameter $4 \mathrm{~mm}$ (post growth), one for each seed. The composition of each cylindrical buffer layer of dimensions $5 \mathrm{~mm}$ in diameter and $2 \mathrm{~mm}$ in height prior to melt processing was $75 \mathrm{wt} \%$ Y-123 + 25 wt\%Y-211 for each sample. These three samples, which were labelled as samples 1,2 and 3, respectively, were used to investigate the single grain microstructure through the cross-section of the single grain and to measure the superconducting critical transition temperature $\left(T_{\mathrm{c}}\right)$ and critical current density $\left(J_{\mathrm{c}}\right)$ in the vicinity of the seed. The samples were cut initially in half using a diamond saw. One half from each sample was polished to observe the microstructure under an optical microscope. The other halves of samples 1 and 2 were cut into slabs of thickness $1.5 \mathrm{~mm}$ parallel to the single grain cross-section and then cut and cleaved further into smaller sub-specimens of dimensions $a \times b \times c \approx 2.0 \times 1.5 \times 1.0 \mathrm{~mm}^{3}$, as shown in figure 2(c). The magnetic moment of these subspecimens was measured using a superconducting quantum interference device with the $a b$ plane of each sample oriented perpendicular to the applied field.

The extended Bean model [23] was used to estimate $J_{\mathrm{c}}$ for the sub-specimens in the vicinity of the seed. In addition, a set of larger samples (labelled 4-8) of diameter $25 \mathrm{~mm}$ (post growth) were fabricated using a single seed and buffer layers of different diameter to investigate the effects of buffer on trapped field, as shown in (figure 2(a)). The heating profiles for fabricating the single grains were similar, as shown in figure 2(b), with a cooling rate of $0.5{ }^{\circ} \mathrm{Ch}^{-1}$ for samples of diameter $16 \mathrm{~mm}$ and a slower cooling rate $0.3^{\circ} \mathrm{Ch}^{-1}$ for the larger samples, respectively. Trapped fields were measured following a field cooled process, which involved applying a magnetic field of $1.3 \mathrm{~T}$, cooling the sample to $77 \mathrm{~K}$ using liquid nitrogen and then removing the magnetic field. The resulting trapped field was measured using an array of 19 rotating probes with a distance of about $3.5 \mathrm{~mm}$ between each probe. The distance between the probes and the surface of the sample was approximately $0.7 \mathrm{~mm}$.

\section{Results and discussion}

\subsection{Growth sector size}

Figure 3 shows optical micrographs of the cross-sections of samples 1,2 and 3 . The boundaries between the a- and c-growth sectors can be seen clearly in the figure. The diagram beneath each photograph indicates schematically the position of the growth sector boundaries (red dashed lines). It can be seen by comparing the micrographs for samples 1 and 2 that the use of a buffer limits the extent of the a-growth sector at the centre of the sample, with the size of the a-sector correlating inversely with buffer layer diameter over a range of sample size. It has been reported that the bigger the seed the smaller the a-growth sector [24]. Figure 3, therefore, suggests that the buffer layers themselves act as seeds. In the case of the multi-seeded sample (sample 3), the presence of the buffer layers helps reduce the vertical extent of the a-growth sector, which, significantly, is the location of the grain boundary [22]. This implies that the buffering technique can be used effectively to reduce the extent of the grain boundaries in multi-seeded samples. It is understood that the a-/c-sector constitution of the growth boundaries in single YBCO grains, which reflects the different growth rates along 

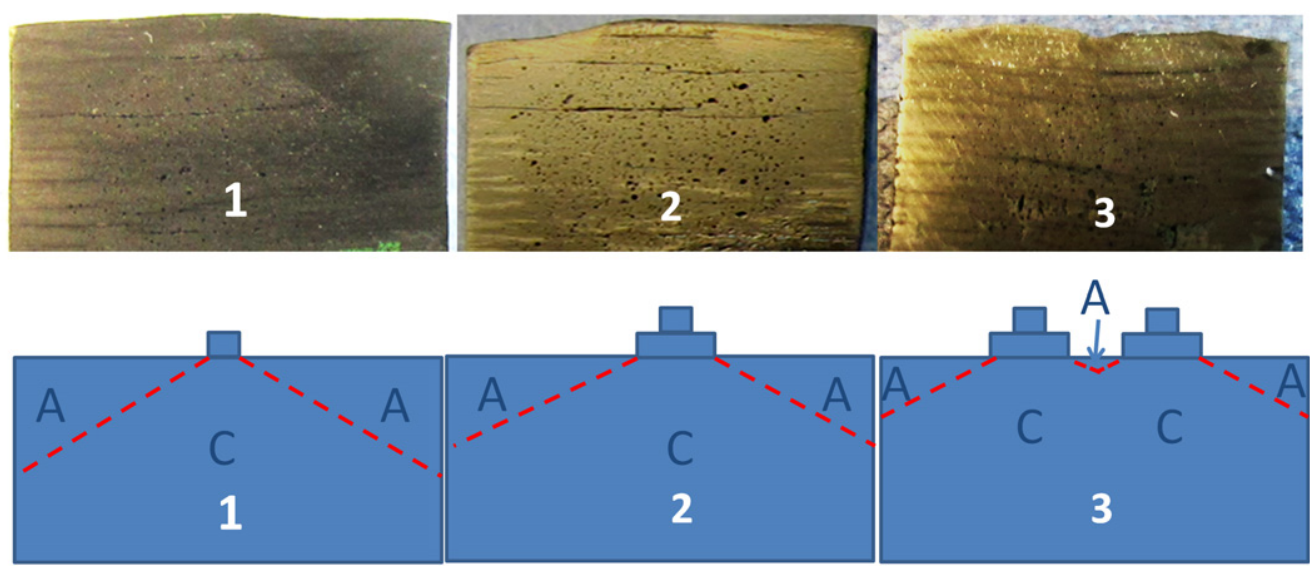

Figure 3. Photographs and corresponding schematic illustrations of the cross-sections of the single grain YBCO samples 1-3.

the $a$ and $c$ lattice directions, can also be influenced by the heating profile and temperature distribution within the furnace. Samples 1, 2 and 3 in the current study were fabricated in the same furnace using same heating profile. Hence, the changes in the relative extent of a-/c-sectors in the growth boundaries appear to be influenced significantly by the use of a buffer layer. The a- and c-sector growth boundaries in figure 3 also indicate directly that the size of the buffer correlates with the size of the seed. The buffer layer, therefore, acts essentially as seed.

\subsection{Equivalence to hot seeding}

The physical requirements for the seeds in the hot seeding process are a melting temperature of at least $T_{\mathrm{g} 1}$ and a similar lattice parameter to that of the RE-123 bulk sample to be seeded. The composition of the buffer layer for all (RE)BCO$\mathrm{Ag}$ systems, therefore, must fulfil the requirements of the seeds for hot-seeding, with the melting temperatures of the buffer layers being decided by its composition. As a result, this is chosen to be the same as that of the pellet to be seeded, but without any dopants and based on previous studies of optimised buffer aided growth of the (RE)BCO-Ag system [17-19]. For example, the buffer layer composition for pellets of composition of $75 \mathrm{wt} \% \quad \mathrm{Sm}-123+25 \mathrm{wt} \% \quad \mathrm{Sm}-$ $211+2 \mathrm{wt} \% \quad \mathrm{BaO}_{2}+1 \mathrm{wt} \% \quad \mathrm{CeO}_{2}+10 \mathrm{wt} \% \quad \mathrm{AgO}_{2}$ is $75 \mathrm{wt} \% \mathrm{Sm}-123+25 \mathrm{wt} \% \mathrm{Sm}-211$. The melting temperature of the buffer layer in this case is $1070{ }^{\circ} \mathrm{C}$, compared to a melting temperature of the seeded pellet of $1030^{\circ} \mathrm{C}$. In general, the melting temperatures of the buffer layers used for (RE)BCO-Ag systems are at least as high as the melting temperatures ( $T_{\mathrm{g} 1}$ in theory) of the RE-123 composition to be seeded due to the fact that any dopant (other than $\mathrm{MgO}$ ) in the precursor powder would reduce its melting temperature, which is clearly not the case.

The sizes of the buffer layers used to aid the TSMG process are usually between 2 and $4 \mathrm{~mm}$ in diameter and between 2 and $3 \mathrm{~mm}$ in height. The role of the buffer is not only to prevent 'harmful' elements such as $\mathrm{Ag}$ diffusing from the growing pellet into the seed, but also, conversely, to inhibit contamination of the growing pellet by the seed
$[10-14,18]$. Buffer layers can, themselves, even grow into small single grains that can be seen clearly by the naked eye during the fast cooling process at a temperature between $T_{\mathrm{m}}$ and $T_{\mathrm{g} 1}$ and prior to the slow cooling process [17, 18]. Importantly, this small, single grain later serves as a seed during the subsequent slow cooling as discussed above, which is directly analogous to the 'hot seeding' [12] process (where a seed is placed on the top surface of the sample at a high temperature between $T_{\mathrm{m}}$ and $T_{\mathrm{g} 1}$ to order to overcome the difficulties of sourcing a seeds with sufficiently high melting temperature).

The buffer technique, which is well known generally in the field of crystal growth, effectively stabilizes the seed and filters impurities from the seeding and melt growth processes $[25,26]$. This is obviously true for the melt processing of (RE)BCO-Ag single grains. It is also believed that there is a mismatch in lattice parameters between the seed and the RE-123 phase of the growing pellet. For example, in the case of using SmBCO seeds to grow YBCO, the lattice parameters $a, b, c$ of the unit cell of the tetragonal Y-123 phase are $3.857,3.857$, and $11.839 \AA$, respectively [39-1496 JCPDS international Centre for Diffraction], whereas the equivalent lattice parameters for tetragonal Sm123 are 3.892, 3.892 and $11.625 \AA$ [45-0415 JCPDS international Centre for Diffraction]. The cumulative effect of this mismatch could extend to several hundred micrometres in the area beneath the seed, which might result in the misarrangement of the atoms during the process of forming the single grain, causing the seeding process to fail [17]. However, the buffer layer, which has the same composition as the seeded pellet but without dopants, has the same lattice parameter as the RE-123 phase in the growing pellet. As a result, the mismatch between the seed and the buffer layer is reduced substantially under air due to the small size of the crystallised buffer layer (i.e. $4-2 \mathrm{~mm}$ in diameter). As a result, the buffer can act potentially as a perfect single crystal (single grain), a significantly improved seed and therefore help the single grain to grow uniformly into a large size. 


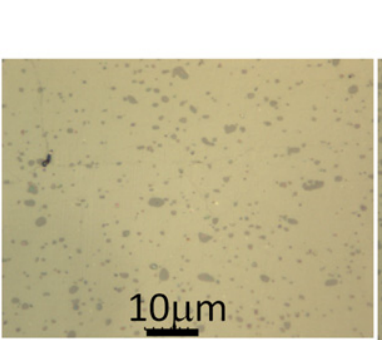

$1 \mathrm{a}$

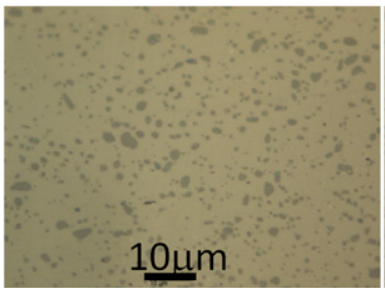

$1 \mathrm{a}$

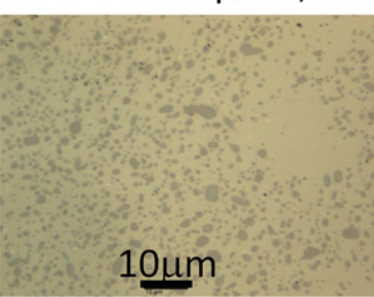

3a

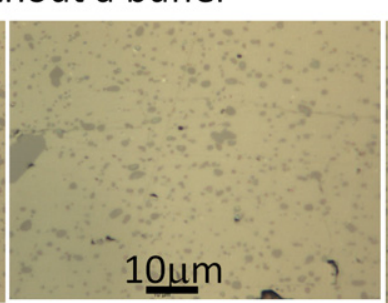

$1 b$

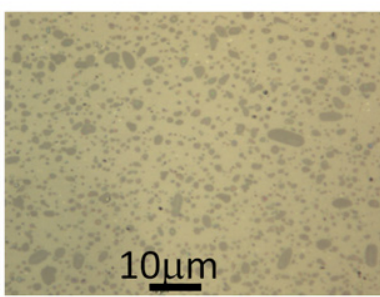

$1 c$

Sample 2, with a buffer

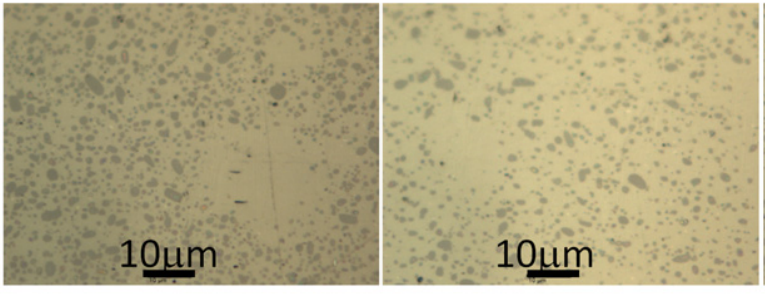

$1 b$

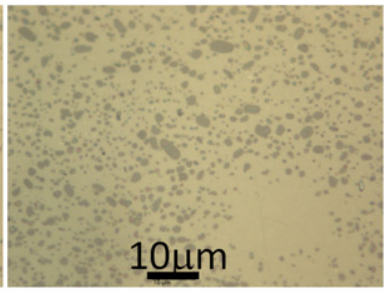

$1 \mathrm{c}$

Figure 4. Microstructures of the sample fabricated with and without buffer layers at the positions $1 \mathrm{a}, 3 \mathrm{a}, 1 \mathrm{~b}$ and $1 \mathrm{c}$ of samples 1 and 2 .
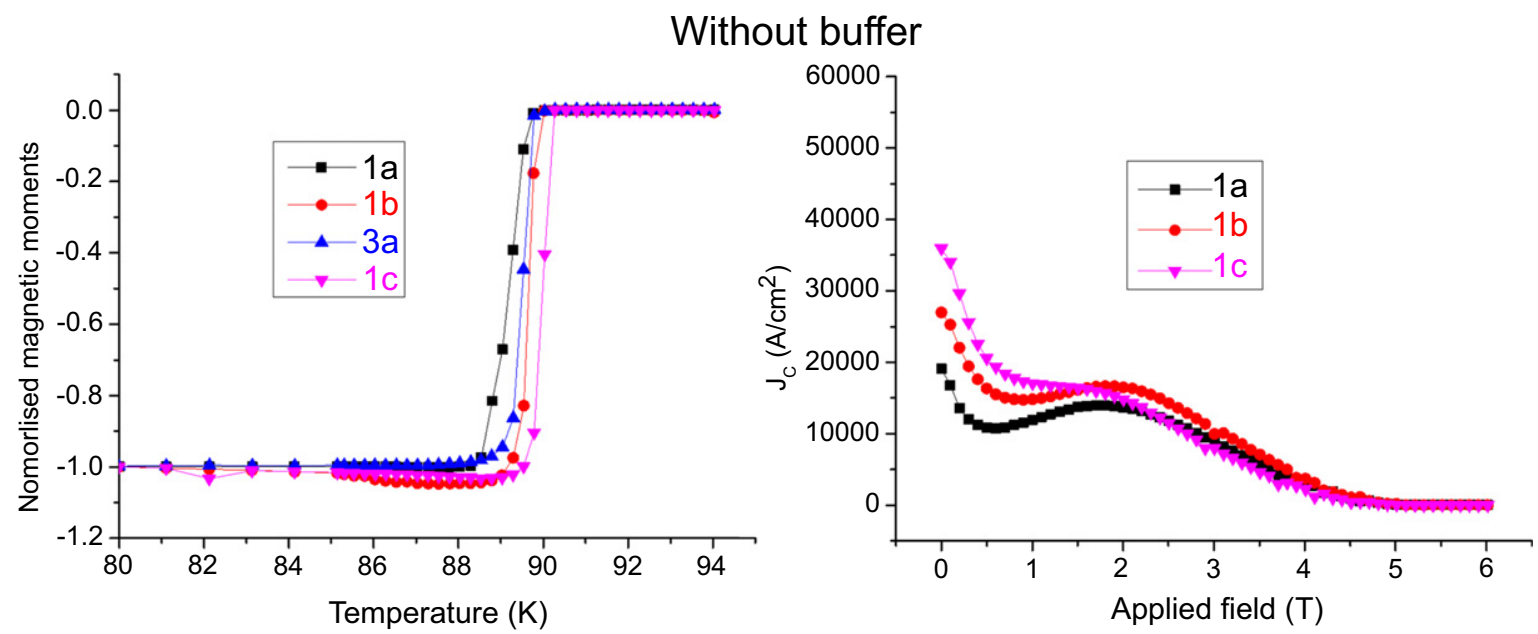

With buffer
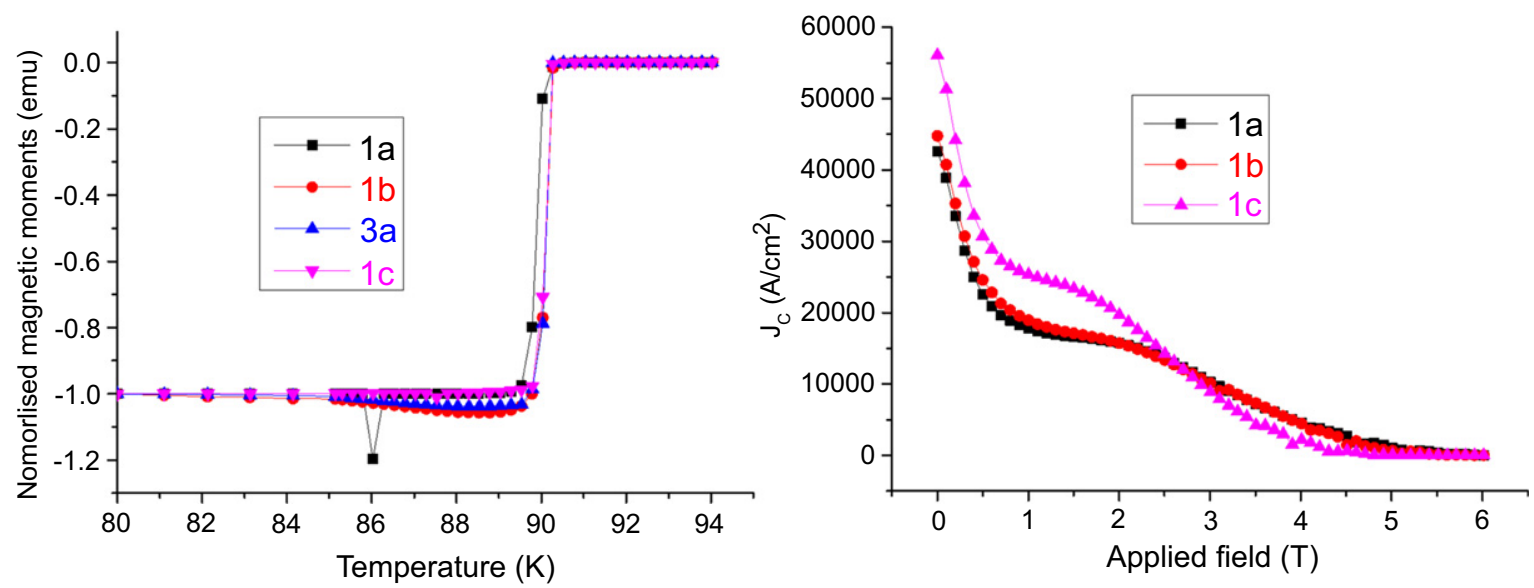

Figure 5. $T_{\mathrm{c}}$ and $J_{\mathrm{c}}$ of the specimens at different positions of the single grain samples fabricated with and without a buffer layer. 

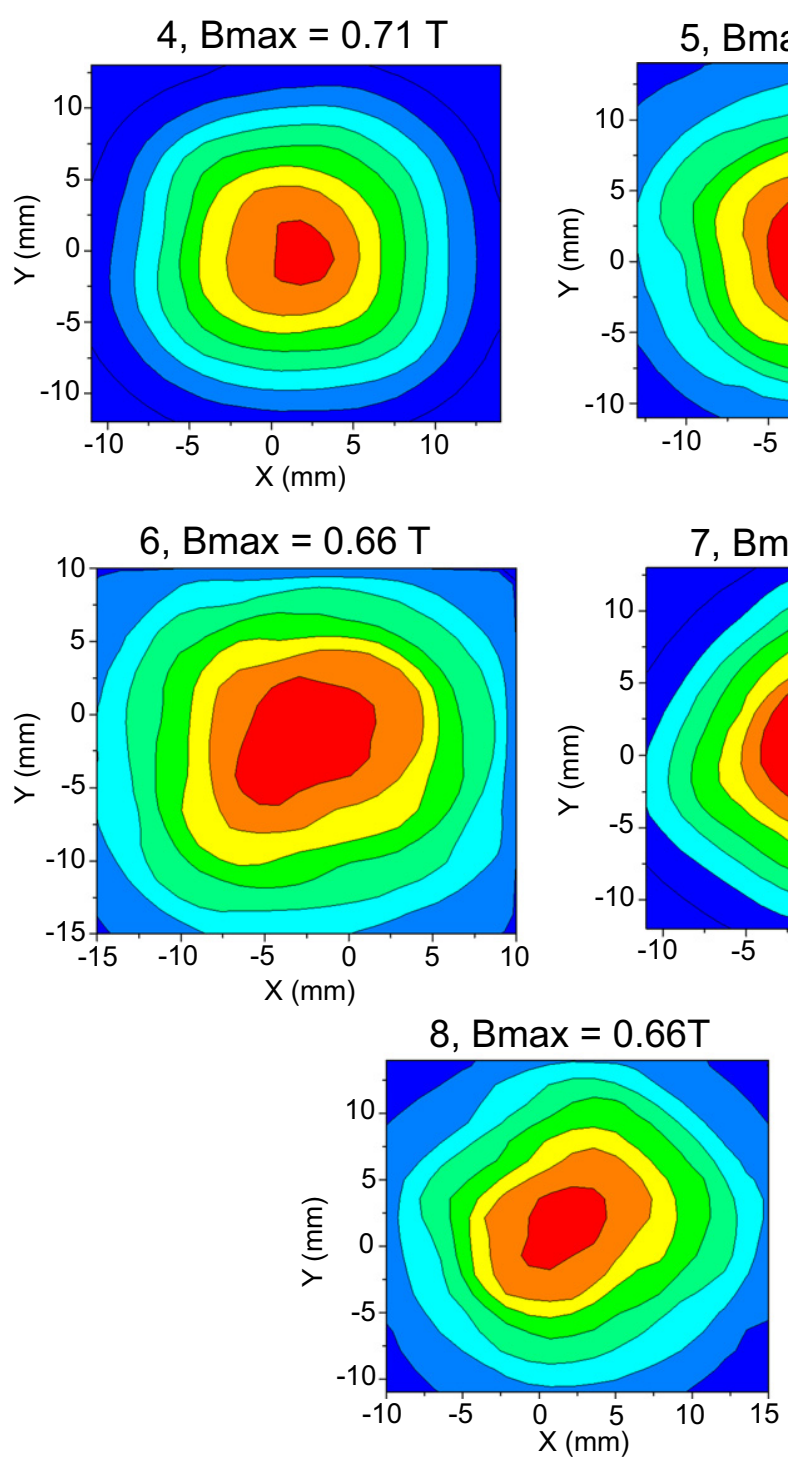
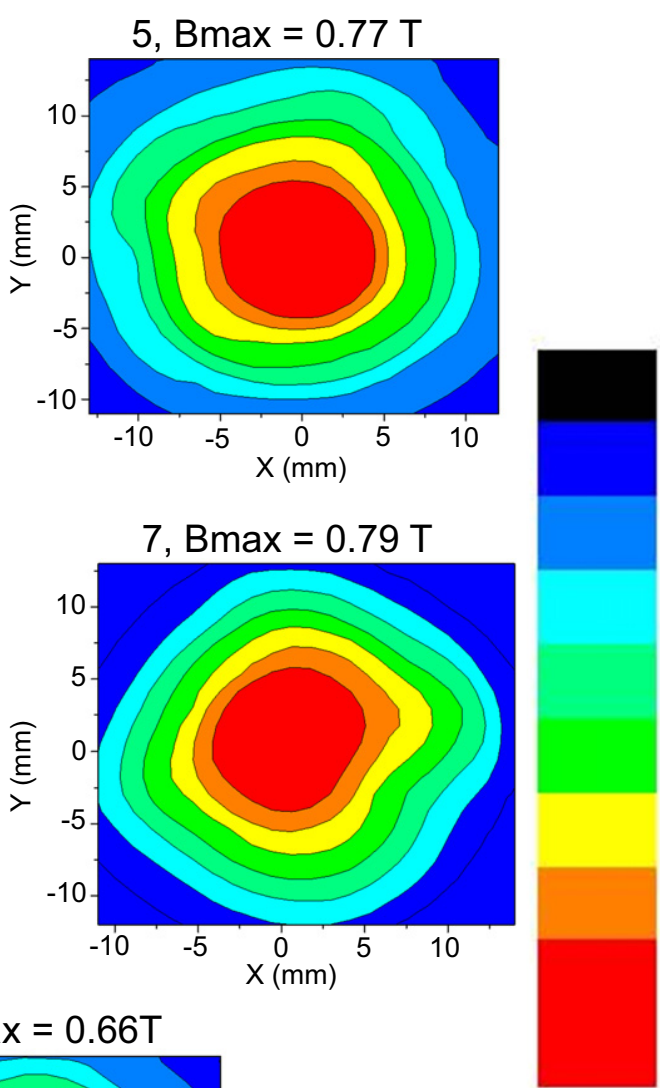
$-0.01$

0.09

0.18

0.28

0.37

0.46

0.56

Figure 6. Trapped field distributions of the single grains grown using buffer layers of different sizes.

\subsection{Buffers change the distribution of RE-211 close to the seed}

Figure 4 shows the microstructures of samples 1 and 2 at positions $1 \mathrm{a}, 1 \mathrm{~b}, 1 \mathrm{c}$ and $3 \mathrm{a}$, which correspond to those used to calculate $J_{\mathrm{c}}$ (see figure 2(c)). It can be seen that the concentration of Y-211 particles at position 1a (i.e. that immediately beneath the seed) in the sample grown without a buffer layer is the lowest of all the positions. This is expected since the Y-211 particles react with the liquid phase to form the $\mathrm{Y}-123$ phase more extensively under conditions of low under-cooling [27]. It should be noted that this Y-211 concentration is also lower than that at position 1a in the samples fabricated with a buffer layer. The concentration of the Y-211 particles at position $1 \mathrm{~b}$ in sample 1 is slightly lower than that in sample 2. However, samples 1 and 2 exhibit similar concentrations of the Y-211 particles at positions $3 \mathrm{a}$ and $1 \mathrm{c}$. This observation indicates that influence of the buffer layer on the Y-211 distribution is only obvious in the area in the vicinity of the seed and within roughly a radius of $3 \mathrm{~mm}$ (position 1a is approximately 2-3 $\mathrm{mm}$ from position $3 \mathrm{a}$ ). In other words, when a buffer layer is used, the concentration of Y-211 in the sample will be higher at a position close to the seed because the 1a position in the sample fabricated with a buffer layer is more distant from the seed. This sample would therefore contain more Y-211 particles at positions more distant from the seed due to the associated higher growth rate [27] according to the results from image analysis, and according to trapping/pushing phenomenon [28]. Of course, the distribution of the Y-211 phase can also be affected by other factors, such as precursor powder composition, heating profile and the temperature distribution within the furnace. However, given that samples 1 and 2 were grown using the same precursor powder and in the same furnace at the same time, it may be concluded that the differences in the Y-211 distribution between samples 1 and 2 are attributed to the use of a buffer layer. 
3.4. The improvement of superconducting properties $T_{c}$ and $J_{C}$ of the position under the buffers

Figure 5 shows $T_{\mathrm{c}}$ and $J_{\mathrm{c}}$ of the specimens at positions $1 \mathrm{a}, 1 \mathrm{~b}$, $1 \mathrm{c}$ and $3 \mathrm{a}$ of samples 1 and 2 . It can be seen that only the $T_{\mathrm{c}}$ at position 1a of sample 1, which is the sample grown without a buffer, is lower and broader. The other specimens all exhibit the same $T_{\mathrm{c}}$, of $90 \mathrm{~K}$ and a sharp superconducting transition. The broad $T_{\mathrm{c}}$ at position $1 \mathrm{a}$ is due to $\mathrm{Nd}$ contamination of the Y-123 phase by the seed $[12,13]$, which extends to a depth of roughly $1 \mathrm{~mm}$ [18]. As a result of the use of a buffer layer, however, the specimens from sample 2 all exhibit the same $T_{\mathrm{c}}$ and a sharp transition width, which suggests that the $\mathrm{Nd}$ contamination from the seed is reduced for these samples. Most importantly, the values of $J_{\mathrm{c}}$ observed for sample 2 fabricated with a buffer layer are higher than those observed in sample 1, which was prepared without a buffer. This is because the use of the buffer layer has increased the Y-211 content at position 1a due to the increase in effective distance from the seed. A larger concentration of Y-211 results generally in a higher concentration of pinning centres, and hence higher $J_{c}$, as has been reported by many researchers [29-31].

Figure 6 shows the measured trapped fields of samples 4-8. It can be seen that there is a small increase in trapped field when the average values observed for samples 5, 6 and 7 (i.e. those fabricated with a buffer layer) of $0.74 \pm 0.05 \mathrm{~T}$ are compared to that observed for sample 1 (the sample without buffer) of $0.71 \mathrm{~T}$. However, the value of trapped field measured for sample $4(0.71 \mathrm{~T})$ falls within the range of the trapped fields measured for the samples fabricated using a buffer. The trapped field of sample 8 , which used a buffer layer of diameter $8 \mathrm{~mm}$, is lower than sample 4 , although a crack is clearly present in the top surface of this sample (figure 2). (The lower trapped field of sample 8 relative to that observed in other samples may be due to the crack at the top surface.) The trapped field was anticipated theoretically to increase slightly for this sample due to the increase in the pinning centre density at the centre of the top surface, assuming a uniform single grain microstructure, other than the Y-211 distribution $[32,33]$. However, the value of trapped field can also be influenced by other defects, such as cracks and pores, which occur randomly throughout the single grain sample. The shapes of the trapped field profiles in figure 6 may also suggest that the samples fabricated in this study are non-uniform. Therefore, it can be concluded that the trapped fields observed for the single grains fabricated using a buffer layer remain improved slightly or unchanged due to this nonuniformity. More research is needed to improve the uniformity of the single grain materials fabricated by the buffer layer technique, and this is the subject of an on-going investigation.

\section{Conclusions}

The newly developed buffer layer technique is a general modification to the melt processing method that can be used in the primary growth, recycling process and even infiltration growth of all (RE)BCO-Ag systems. The use of a buffer layer enhances significantly the reliability of the seeding step, which is critical to the melt process.

We have demonstrated that buffer layers function as improved seeds by providing process advantages that are inherent to the hot seeding melt processing technique. The buffer layers act essentially as 'pseudo hot seeds' in this process, resulting in a decrease in the relative proportion of a-/c-sectors in the single grain microstructure depending on the size of the buffer. Consequently, the local distribution of RE-211 under the position of the buffer layer is increased, as is the local $J_{\mathrm{c}}$. The $T_{\mathrm{c}}$ in the vicinity of the seed both increases and becomes shaper due to a reduced contamination from the seed for single grain samples fabricated using a buffer layer. The peak trapped field of these samples remains unchanged or improves slightly, which is potentially significant for the manufacture of bulk single grain superconductors for practical applications.

\section{Acknowledgments}

This work was supported by the Engineering and Physical Sciences Research Council (EPSRC, grant number EP/ K02910X/1) and King Abdulaziz City for Science and Technology (KACST). Additional data related to this publication are available at the University of Cambridge data repository (https://www.repository.cam.ac.uk/handle/1810/ 249091). All other data accompanying this publication are directly available within the publication.

\section{References}

[1] Hull J R 2000 Supercond. Sci. Technol. 13 R1

[2] Koshizuka N 2006 Physica C 445-448 1103

[3] Koch R, Wagner R, Sander M and Gutt H J 2000 J. Phys.: Conf. Ser. 1671055

[4] Durrell J H et al 2014 Supercond. Sci. Technol. 27082001

[5] Todt V R, Zhang X F, Miller D J, St Louis-Weber M and Dravid V P 1996 Appl. Phys. Lett. 69 3746-8

[6] Cardwell D A 1998 J. Mater. Sci. Eng. B 53 1-10

[7] Shi Y, Hari Babu N and Cardwell D A 2005 Supercond. Sci. Technol. 18 L13-6

[8] Nariki S, Sakai N and Murakami M 2005 Supercond. Sci. Technol. 18 S126

[9] Shi Y, Babu N H, Iida K, Yeoh W K, Dennis A R, Pathak S K and Cardwell D A 2010 Physica C 470685

[10] Li T Y, Cheng L, Yan S B, Sun L J, Yao X, Yoshida Y and Ikuta H 2010 Supercond. Sci. Technol. 23125002

[11] Oda M, Yao X, Yoshida Y and Ikuta H 2009 Supercond. Sci. Technol. 22075012

[12] Chauhan H S and Murakami M 2000 Supercond. Sci. Technol. 13 672-5

[13] Kim C-J, Kim H-J, Jee Y-A, Hong G-W, Joo J-H, Han S-C, Han Y-H, Sung T-H and Kim S-J 2000 Physica C 338205

[14] Kim C-J, Lee J H, Park S-D, Jun B-H, Han S C and Han Y H 2011 Supercond. Sci. Technol. 24015008

[15] Xu H H, Chen Y Y, Cheng L, Yab S B, Yu D Y and Yao X 2012 Supercond. Sci. Technol. 25035014

[16] Zhou D-F, Xu K, Hara S, Li B-Z, Deng Z, Tsuzuki K and Izumi M 2012 Supercond. Sci. Technol. 25025022 
[17] Shi Y-H, Dennis A R and Cardwell D A 2015 Supercond. Sci. Technol. 28035014

[18] Namburi D K, Shi Y, Zhai W, Dennis A R, Durrell J H and Cardwell D A 2015 Cryst. Growth Des. 15 1472-80

[19] Zhao W, Shi Y, Dennis A R and Cardwell David A 2015 IEEE Trans. Appl. Supercond. 255801305

[20] Shi Y, Namburi D K, Wang M, Durrell J, Dennis A and Cardwell D 2015 accepted by J. Am. Ceram. Soc. 98 2760-6

[21] Namburi D K, Shi Y, Dennis A R, Durrell J H and Cardwell D A 2015 J. Eur. Ceram. Soc. in press

[22] Shi Y, Durrell J H, Dennis A R, Nadendla H B, Mancini C E and Cardwell D A 2012 Supercond. Sci. Technol. 25045006

[23] Chen D-X and Goldfarb R B 1989 J. Appl. Phys. 662489

[24] Li H-C, Fan W-S, Peng B-N, Wang W, Zhuang Y-F, Guo L-S, Yao X and Ikuta H 2015 Cryst. Growth Des. 15 1740-4
[25] Bergfors T 2003 J. Struct. Biol. 14266

[26] Stewart P D S, Kolek S A, Briggs R A, Chayen N E and Baldock P F M 2011 Cryst. Growth Des. 113432

[27] Zhai W, Shi Y, Durrell J H, Dennis A R and Cardwell D A 2014 Cryst. Growth Des. 14 6367-75

[28] Endo A, Chauhan H S, Egi T and Shiohara Y 1996 J. Mater. Res. 11795

[29] Nariki S, Seo S J, Sakai N and Murakami M 2000 Supercond. Sci. Technol. 13 778-84

[30] Nariki S, Sakai N, Murakami M and Hirabayashi I 2004 Physica C 412-414 557-65

[31] Cloots R, Koutzarova T, Mathieu J P and Ausloos M 2005 Supercond. Sci. Technol. 18 R9-23

[32] Zhai W, Shi Y, Durrell J H, Dennis A R, Zhang Z and Cardwell D A 2015 Cryst. Growth Des. 15 907-14

[33] Zou J, Ainslie M D, Hu D, Zhai W, Namburi D K, Durrell J H, Shi Y-H and Cardwell D A 2015 Supercond. Sci. Technol. 28035016 